\title{
Hyperdeterminantal calculations of Selberg's and Aomoto's integrals
}

\author{
Jean-Gabriel Luque $^{1} \quad$ Jean-Yves Thibon ${ }^{2}$ \\ Dedicated to the Memory of Brian G. Wybourne
}

${ }^{1}$ IGM, Université de Marne-la-Vallée, 77454 Marne-la-Vallée Cedex 2, France (luque@univ-mlv.fr)

${ }^{2}$ IGM, Université de Marne-la-Vallée, 77454 Marne-la-Vallée Cedex 2, France (jyt@univ-mlv.fr) 


\begin{abstract}
The hyperdeteminants considered here are the simplest analogues of determinants for higher rank tensors which have been defined by Cayley, and apply only to tensors with an even number of indices. We have shown in a previous article that the calculation of certain multidimensional integrals could be reduced to the evaluation of hyperdeterminants of Hankel type. Here, we carry out this computation by purely algebraic means in the cases of Selberg's and Aomoto's integrals.
\end{abstract}




\section{Introduction}

Selberg's integral and its generalizations are multiple integrals encountered in many fields such as mathematical statistics, random matrices, statistical mechanics, special functions, integrable systems, and can be interpreted as generalized moments, correlation functions or partition functions.

Such integrals appear naturally in the computation of the normalization constants for various random matrix ensembles. For example the normalization of the eigenvalue probability density function for Gaussian, circular, Laguerre, Jacobi and Cauchy ensembles are limit cases of Selberg integrals (see [31, 14, 15]). Generalizations appear in the calculation of the partition functions of log-potential Coulomb systems [16]. Such multiple moment integrals are also connected with the problem of expanding even powers of the Vandermonde determinant

$$
\Delta(x)=\operatorname{det}\left(x_{i}^{j-1}\right)=\prod_{1 \leq i<j i \leq n}\left(x_{j}-x_{i}\right)
$$

in various bases of symmetric functions. Expansion of these powers in terms of Schur functions is still an open problem (see [32, 39, 21]), whose solution would find important applications to calculations related to some aspects of the fractional quantum Hall effect (see [32, 40]) described by Laughlin's wave function 26]

$$
\Psi^{m}\left(z_{1}, \ldots, z_{n}\right)=\prod_{i<j}^{N}\left(z_{i}-z_{j}\right)^{2 m+1} \exp \left\{-\frac{1}{2} \sum_{i=1}^{N}\left|z_{i}\right|^{2}\right\}
$$

The same expansion would allow one to compute many more interesting integrals (see [14, 16, 27, 28]).

Selberg's integral is a generalization of Euler's Beta integral, introduced by himself in 1944 (see [33]), in view of proving a generalization of a theorem of Gelfond. Selberg found that his integral could be written as a product of quotients of gamma functions

$$
\begin{aligned}
\mathcal{S}_{n}(a, b ; \gamma) & =\int_{0}^{1} \cdots \int_{0}^{1}|\Delta(x)|^{2 \gamma} \prod_{i=1}^{n} x_{i}^{a-1}\left(1-x_{i}\right)^{b-1} d x_{i} \\
& =\prod_{j=0}^{n-1} \frac{\Gamma(a+j \gamma) \Gamma(b+j \gamma) \Gamma((j+1) \gamma+1)}{\Gamma(a+b+(n+j-1) \gamma) \Gamma(\gamma+1)} .
\end{aligned}
$$

This identity was subsequently proved in many different ways by other authors, such as Anderson [1], Aomoto [3] and Dotsenko and Fateev [12. These 
proofs are all interesting, as they involve generalizations of Selberg's integral or techniques which can be useful in other contexts. However, Selberg's original proof has the specificity that it is almost elementary, in the sense that he computed the integral in the case where $\gamma$ is an integer $k$, using only arguments on the degrees of the polynomials appearing after integration of the terms obtained by expansion of $\Delta(x)^{2 k}$, and a very simple change of variables. Then, he concluded by involving a classical theorem of Carlson to extend it to complex values of $\gamma$ (see [31] or [36]). As we shall see below, the elementary part of this proof comes out naturally if we consider this object for $\gamma=k \in \mathbb{N}$ not as an integral but as a special case of a hyperdeterminant, a notion originally due to Cayley but whose only available textbook presentations seem to be Refs. [34, 35] (in Russian).

The hyperdeterminant is a polynomial invariant of tensors of even order (i.e., with an even number of indices). In the case of matrices, its definition gives back the classical determinant.

We have explained in 28] that Selberg's integral could be rewritten as a hyperdeterminant of Hankel type (i.e., whose entries depend only on the sum of the indices) built from the moments of the beta distribution. In this paper, we give a complete translation of Selberg's proof in the hyperdeterminantal formalism, using only very simple tools. The hyperdeterminantal aspects of multiple integral computations look very promising. At present, we know only a few tools to handle such polynomials, but this is already sufficient to deal with some interesting cases. To illustrate this point, we sketch a hyperdeterminantal proof of Aomoto's integral

$$
\begin{aligned}
\mathcal{A}_{n}^{a, b ; k}(y) & =\int_{0}^{1} \cdots \int_{0}^{1} \Delta(x)^{2 k} \prod_{i=1}^{n}\left(y-x_{i}\right) x_{i}^{a-1}\left(1-x_{i}\right)^{b-1} d x_{1} \cdots d x_{n} \\
& =(-2)^{-n} S_{n}(a, b ; k) P_{n}^{\frac{a}{k}-1, \frac{b}{k}-1}(1-2 y)
\end{aligned}
$$

where $P_{n}^{a, b}$ are the monic Jacobi polynomials. Aomoto has proved this identity in [3] by means of differential equations. The main interest of Aomoto's original proof is its relation with the Calogero model. Barsky and Carpentier have given in 4] a different proof, based on Anderson's method.

\section{Elementary hyperdeterminant calculus}

\subsection{Definition}

We shall find it convenient to use a set of $n$ Grassmann (that is, anticommutative, or fermionic) variables $\eta=\left\{\eta_{1}, \ldots, \eta_{i}, \ldots\right\}$. A tensor of order $k$ 
and dimension $n$, that is, an element of $V^{\otimes k}$, where $V$ is an $n$ dimensional vector space, will be represented as

$$
\mathbf{M}=\sum_{1 \leq i_{1}, \cdots, i_{k} \leq n} M_{i_{1} \cdots i_{k}} \eta_{i_{1}} \otimes \cdots \otimes \eta_{i_{k}} .
$$

That is, we identify $V$ with the vector space spanned by $\eta_{1}, \ldots, \eta_{n}$. The array $\left(M_{i_{1} \ldots i_{k}}\right)$ will be called a hypermatrix. The hyperdeterminant $\operatorname{Det}(\mathbf{M})$ of $\mathbf{M}$ can be compactly defined as the coefficient of $\left(\eta_{1} \cdots \eta_{n}\right)^{\otimes k}$ in $\frac{\mathbf{M}^{n}}{n !}$ :

$$
\mathbf{M}^{n}=n ! \operatorname{Det}(\mathbf{M})\left(\eta_{1} \cdots \eta_{n}\right)^{\otimes k}
$$

where the power $\mathbf{M}^{n}$ is evaluated in the tensor product of $k$ copies of the Grassmann algebra over the $n$ distinct $\eta_{i}$ 's, so that the only surviving terms are those involving $\left(\eta_{1} \cdots \eta_{n}\right)^{\otimes k}$.

For convenience, we will use the notation

$$
\operatorname{Det}_{k}\left(M_{i_{1} \cdots i_{k}}\right)_{1}^{n}=\operatorname{Det}(\mathbf{M}) \text {. }
$$

Note first that if $\mathbf{M}$ is a tensor of odd order $k$, one has $\operatorname{Det}(\mathbf{M})=0$. If $\mathbf{M}$ is a tensor of even order $2 k$ and dimension $n$, one has

$$
\operatorname{Det}(\mathbf{M})=\frac{1}{n !} \sum_{\sigma_{1}, \cdots, \sigma_{2 k} \in \mathfrak{S}_{n}} \epsilon\left(\sigma_{1}\right) \cdots \epsilon\left(\sigma_{2 k}\right) \prod_{i=1}^{n} M_{\sigma_{1}(i) \ldots \sigma_{2 k}(i)}
$$

Setting $k=1$, we recover the classical definition of the determinant for matrices.

\subsection{The invariance property}

Definition (7) is very useful for proving properties of hyperdeterminants. Let us consider the natural action of the group $G L(V)^{\times 2 k}$ on $V^{\otimes 2 k}$

$$
\left(g^{(1)} \otimes \cdots \otimes g^{(2 k)}\right) \cdot \mathbf{v}_{1} \otimes \cdots \otimes \mathbf{v}_{2 k}=\left(g^{(1)} \mathbf{v}_{1}\right) \otimes \cdots \otimes\left(g^{(2 k)} \mathbf{v}_{2 k}\right)
$$

Then, for a general $\mathbf{M} \in V^{\otimes 2 k}$,

$$
\begin{aligned}
\left(g^{(1)} \otimes \cdots\right. & \left.\otimes g^{(2 k)}\right) \cdot \mathbf{M}=\sum_{1 \leq j_{1}, \cdots, j_{2 k} \leq n} M_{j_{1} \cdots j_{2 k}} \times \\
& \times \sum_{i_{1}=1}^{n} g_{i_{1} j_{1}}^{(1)} \eta_{j_{1}} \otimes \cdots \otimes \sum_{i_{2 k}=1}^{n} g_{i_{2 k} j_{2 k}}^{(2 k)} \eta_{j_{2 k}} .
\end{aligned}
$$

Hence,

$$
\operatorname{Det}\left(\left(g^{(1)} \otimes \cdots \otimes g^{(2 k)}\right) \cdot \mathbf{M}\right)=\operatorname{det}\left(g^{(1)}\right) \cdots \operatorname{det}\left(g^{(2 k)}\right) \operatorname{Det}(\mathbf{M}) .
$$




\subsection{Minor summation formula}

Consider two tensors

$$
\mathbf{M}=\sum_{1, \leq i_{1} \ldots, i_{2 k} \leq n} M_{i_{1} \ldots i_{2 k}} \eta_{i_{1}} \otimes \cdots \otimes \eta_{i_{2 k}}
$$

and

$$
\mathbf{N}=\sum_{1 \leq i_{1}, \ldots, i_{2 k} \leq n} N_{i_{1} \ldots i_{2 k}} \eta_{i_{1}} \otimes \cdots \otimes \eta_{i_{2 k}} .
$$

Remarking that $\mathbf{M N}-\mathbf{N M}=0$ (in the $2 k$ th tensor power of the Grassmann algebra), one has

$$
(\mathbf{M}+\mathbf{N})^{n}=\sum_{i=0}^{n}\left(\begin{array}{l}
n \\
i
\end{array}\right) \mathbf{M}^{i} \mathbf{N}^{n-i}
$$

If $I=\left(I_{1}, \cdots, I_{2 k}\right)$ is a $2 k$-tuple of subsets of $\{1, \ldots, n\}$, we will denote by $\mathbf{M}[I]$ the tensor

$$
\mathbf{M}[I]=\sum_{i_{1} \in I_{1}, \ldots, i_{2 k} \in I_{2 k}} M_{i_{1} \cdots i_{2 k}} \eta_{i_{1}} \otimes \cdots \otimes \eta_{i_{2 k}}
$$

and by $\eta_{I}$ the product

$$
\eta_{I}=\overrightarrow{\prod_{i_{1} \in I_{1}}} \eta_{i_{1}} \otimes \cdots \otimes \prod_{i_{2 k} \in I_{2 k}} \eta_{i_{2 k}}
$$

where the symbol $\vec{\prod}_{i}$ denotes the product taken in increasing order of the subscripts.

Let $\mathfrak{C}_{n, k}^{r}$ be the set of pairs $(I, J)$ of $2 k$-tuples $I=\left(I_{1}, \cdots, I_{2 k}\right)$ and $J=\left(J_{1}, \cdots, J_{2 k}\right)$ such that for each $s \in\{1, \ldots, 2 k\},\left(I_{s}, J_{s}\right)$ is a partition of $\{1, \ldots, n\}$ into two blocks of sizes $r$ and $n-r$. For each $(I, J) \in \mathfrak{C}_{n, k}^{r}$ we will denote by $\epsilon(I, J)$ the product of the signs of the permutations $\sigma_{s}=$ $\left(i_{1}, \ldots, i_{r}, j_{1}, \ldots, j_{n}\right)$ (written as a word) where $I_{s}=\left\{i_{1}<\cdots<i_{r}\right\}$ and $J_{s}=\left\{j_{1}<\cdots<j_{n-r}\right\}$.

We can now write, for each $p \leq n$,

$$
\mathbf{M}^{p}=p ! \sum_{I} \operatorname{Det}(\mathbf{M}[I]) \eta_{I}
$$

where the sum is over the $2 k$-tuples $I=\left(I_{1}, \cdots, I_{2 k}\right)$ of subsets of $\{1, \ldots, n\}$ of cardinality $p$. Hence, by (15),

$$
\operatorname{Det}(\mathbf{M}+\mathbf{N})=\sum_{r=0}^{n} \sum_{(I, J) \in \mathfrak{C}_{n, k}^{r}} \epsilon(I, J) \operatorname{Det}(\mathbf{M}[I]) \operatorname{Det}(\mathbf{N}[J])
$$


Now, if we set

$$
\mathbf{M}^{\prime}=\sum_{1 \leq i_{2}, \ldots, i_{2 k} \leq n} M_{1, i_{2}, \ldots, i_{2 k}} \eta_{1} \otimes \eta_{i_{2}} \otimes \cdots \otimes \eta_{i_{2 k}}
$$

and

$$
\mathbf{M}^{\prime \prime}=\sum_{i_{1}=2}^{n} \sum_{1 \leq i_{2}, \ldots, i_{2 k} \leq n} M_{i_{1}, i_{2}, \ldots, i_{2 k}} \eta_{i_{1}} \otimes \cdots \otimes \eta_{i_{2 k}},
$$

remarking that $\mathbf{M}=\mathbf{M}^{\prime}+\mathbf{M}^{\prime \prime}$ we obtain from (19),

$$
\operatorname{Det}(\mathbf{M})=\sum_{I=\left(i_{1}=1 \leq i_{2}, i_{3}, \ldots, i_{2 k} \leq n\right)} \operatorname{sign}(I) M_{1, i_{2}, \ldots, i_{2 k}} \operatorname{Det}(\mathbf{M}[\bar{I}])
$$

where $\operatorname{sign}(I)=(-1)^{i_{1}+i_{2}+\cdots+i_{2 k}}$ and $\bar{I}=\left(\{1, \ldots, n\}-i_{1}, \ldots,\{1, \ldots, n\}-i_{2 k}\right)$. Equations (19) and (22) can be found in [5, 34, 35].

\section{$3 \quad$ Hankel hypermatrices}

For a sequence $I=\left(i_{1}, \ldots, i_{2 k}\right)$, we will set $|I|=i_{1}+\cdots+i_{2 k}$ and $\eta_{I}=$ $\eta_{i_{1}} \otimes \cdots \otimes \eta_{i_{2 k}}$.

\subsection{Heine's integrals for Hankel determinants}

Let $\mu$ be a measure on the real line, and $c_{n}=\int x^{n} d \mu(x)$ be its moments. Heine's integrals below are evaluated as Hankel determinants whose entries are the moments $c_{n}$ (see [19])

$$
\int \cdots \int \Delta(x)^{2} d \mu\left(x_{1}\right) \cdots d \mu\left(x_{n}\right)=n ! \operatorname{det}\left(c_{i+j}\right)_{0}^{n-1} .
$$

It is easy to give a direct proof of this equality, but it can also be seen as a very special case of de Bruijn's integral

$$
\int_{a \leq x_{1}<\cdots<x_{n} \leq b} \cdots \int_{1 e t}\left(\phi_{i}\left(x_{j}\right) \mid \psi_{i}\left(x_{j}\right)\right) d x_{1} \cdots d x_{n}=\operatorname{Pf}\left(Q_{i j}\right)_{1 \leq i, j \leq 2 n}
$$

where $Q_{i j}=\int_{a}^{b}\left[\phi_{i}(x) \psi_{j}(x)-\phi_{j}(x) \psi_{i}(x)\right] d x$ and $\left(\phi_{i}\left(x_{j}\right) \mid \psi_{i}\left(x_{j}\right)\right)$ denotes the matrix whose $i$ th row is $\left[\phi_{i}\left(x_{1}\right), \psi_{i}\left(x_{1}\right), \phi_{i}\left(x_{2}\right), \psi_{i}\left(x_{2}\right), \ldots, \phi_{i}\left(x_{n}\right), \psi_{i}\left(x_{n}\right)\right]$ and $\operatorname{Pf}(M)$ denotes the Pfaffian of the matrix $M$. In [27], we have obtained a generalization of this identity, involving $2 k n$ functions on the left hand side and a hyperpfaffian (see [5]) on the right hand side. In the case where the 
determinant is an even power of the Vandermonde determinant, we obtain a generalization of Heine's theorem turning multiple integrals into Hankel hyperdeterminants. This generalization is discussed in 28 and will be recalled in the following subsection.

\subsection{Generalization: Hankel hyperdeterminants}

We consider here the integral

$$
\mathcal{I}_{n, k}^{\mu}=\int \cdots \int \Delta\left(x_{1}, \cdots, x_{n}\right)^{2 k} d \mu\left(x_{1}\right) \cdots d \mu\left(x_{n}\right)
$$

Expanding the even power of the Vandermonde determinant, this integral can be expressed as a Hankel hyperdeterminant whose entries are the moments $c_{n}=\int x^{n} d \mu(x)$ :

$$
\mathcal{I}_{n, k}^{\mu}=n ! \operatorname{Det}\left(c_{|I|}\right)_{0}^{n-1} .
$$

Consider now the following two tensors

$$
\mathbf{S}_{n}(a, b ; k)=\sum_{0 \leq i_{1}, i_{2}, \ldots, i_{2 k} \leq n-1} \mathrm{~B}(a+|I|, b) \eta_{I}
$$

and

$$
\mathbf{A}_{n}^{a, b ; k}(y)=\sum_{0 \leq i_{1}, i_{2}, \ldots, i_{2 k} \leq n-1} \mathrm{~B}(a+|I|, b) \cdot\left(y-\frac{a+|I|}{a+b+|I|}\right) \cdot \eta_{I},
$$

where $\mathrm{B}(a, b)$ denotes the Beta function $\mathrm{B}(a, b)=\int_{0}^{1} x^{a-1}(1-x)^{b-1} d x$. Remarking that the $\mathrm{B}(a+n, b)$ are the moments of the Beta distribution, one obtains after applying (26)

$$
\mathcal{S}_{n}(a, b ; k)=n ! \operatorname{Det}\left(\mathbf{S}_{n}(a, b ; k)\right)
$$

and in the same way

$$
\mathcal{A}_{n}^{a, b ; k}(y)=n ! \operatorname{Det}\left(\mathbf{A}_{n}^{a, b ; k}(y)\right)
$$

\subsection{Expansion of Hankel hyperdeterminants}

In this section we consider the hyperdeterminant of a general Hankel tensor

$$
\mathcal{H}_{n, k}=\operatorname{Det}_{2 k}\left(X_{i_{1}+\cdots+i_{2 k}}\right)_{0}^{n-1} \text {. }
$$


Expanding this as a polynomial in the $X_{i}$, one finds

$$
\mathcal{H}_{n, k}=\sum_{\lambda} c_{\lambda}^{n, k} X_{\lambda_{1}} \cdots X_{\lambda_{n}}
$$

where the sum is over the $n$-tuples $\lambda=\left(\lambda_{1}, \cdots, \lambda_{n}\right)$ such that $|\lambda|=k n(n-1)$ and such that for each $i \in\{1, \cdots, n\},(i-1) k \leq \lambda_{i} \leq k(n+i-2)$.

This expansion is derived in [28]. A special case appears in Selberg's proof (see 31, 33).

Let $\mathfrak{d}_{n, k}$ be the coefficient of $X_{k(n-1)}^{n}$ in (32):

$$
\mathfrak{d}_{n, k}=c_{(k(n-1))^{n}}
$$

If we set $X_{i}=0$ for $i>k(n-1)$, we obtain from (32) that

$$
\operatorname{Det}_{2 k}\left(X_{i_{1}+\cdots+i_{2 k}}\right)_{0}^{n-1}=\mathfrak{d}_{n, k} X_{k(n-1)}^{n} .
$$

A closed form for $\mathfrak{d}_{n, k}$ follows from a well-known constant term identity (the "Dyson conjecture") ${ }^{1}$

$$
\mathcal{C}_{n, k}=\mathrm{C} . \mathrm{T} .\left\{\prod_{\substack{i, j=1 \\
i \neq j}}^{n}\left(1-\frac{x_{i}}{x_{j}}\right)^{a_{i}}\right\}=\left(\begin{array}{c}
a_{1}+\cdots+a_{n} \\
a_{1}, \cdots, a_{n}
\end{array}\right),
$$

where C.T. means "constant term". On the other hand,

$$
\prod_{\substack{i, j=1 \\ i \neq j}}^{n}\left(1-\frac{x_{i}}{x_{j}}\right)^{k}=(-1)^{k} \Delta(x)^{2 k} \prod_{i=1}^{n} \frac{1}{x_{i}^{k(n-1)}} .
$$

Expanding the power of the Vandermonde determinant, one finds that for $a_{i} \equiv k$,

$$
\begin{aligned}
\mathcal{C}_{n, k} & =(-1)^{k} \mathrm{C} . \mathrm{T} .\left\{\sum_{\sigma_{1}, \cdots, \sigma_{2 k} \in \mathfrak{S}_{n}} \epsilon\left(\sigma_{1}\right) \cdots \epsilon\left(\sigma_{2 k}\right) \prod_{i=1}^{n} x_{i}^{\sigma_{1}(i)+\cdots+\sigma_{2 k}(i)-2 k-k(n-1)}\right\} \\
& =(-1)^{k} n ! \operatorname{Det}_{2 k}\left(\delta_{i_{1}+\cdots+i_{2 k}, n}\right)_{0}^{n-1}
\end{aligned}
$$

where $\delta_{i, j}=1$ if $i=j$ and 0 otherwise (Kronecker symbol). Using (34), we obtain

$$
\mathcal{C}_{n, k}=(-1)^{k} n ! \mathfrak{d}_{n, k}
$$

Hence,

$$
\mathfrak{d}_{n, k}=(-1)^{k} \frac{1}{n !}\left(\begin{array}{c}
k n \\
k, \cdots, k
\end{array}\right) .
$$

\footnotetext{
${ }^{1}$ This was conjectured by Dyson [13 in 1962. I. J. Good gave, in 1970, an elegant elementary proof involving only Lagrange interpolation [18].
} 


\subsection{Minors of a Hankel hyperdeterminant}

We consider here a Hankel hyperdeterminant

$$
\mathbf{M}=\sum_{I} M_{|I|} \eta_{i_{1}} \otimes \cdots \otimes \eta_{i_{2 k}}
$$

and a family of $2 k$ subsets $J=\left(J^{1}, \ldots, J^{2 k}\right)$ of a fixed size $m \leq n$ with $J^{l}=\left\{j_{0}^{l} \leq j_{1}^{l} \leq \cdots \leq j_{m}^{l}\right\} \subset\{0, \ldots, n-1\}$. We want to expand the polynomial $M[J]^{m}$, where

$$
\mathbf{M}[J]=\sum_{I} M_{j_{i_{1}}^{1}+\cdots+j_{i_{2 k}}^{2 k}} \eta_{i_{1}} \otimes \cdots \otimes \eta_{i_{2 k}}
$$

One has

$$
\operatorname{Det}(\mathbf{M}[J])=\frac{1}{n !} \sum_{\sigma_{1}, \ldots, \sigma_{2 k} \in \mathfrak{S}_{m}} \epsilon\left(\sigma_{1}\right) \cdots \epsilon\left(\sigma_{2 k}\right) \prod_{l=1}^{m} M_{j_{\sigma_{1}(l)}^{1}+\cdots+j_{\sigma_{2 k}(l)}^{2 k}} .
$$

But for each $2 k$-tuple $\left(\sigma_{1}, \ldots, \sigma_{2 k}\right)$, one has

$$
\prod_{l=1}^{m} M_{j_{\sigma_{1}(l)}^{1}+\cdots+j_{\sigma_{2 k}(l)}^{2 k}}=\prod_{l=1}^{m} M_{\sigma_{1}(l)+\cdots+\sigma_{2 k}(l)-2 k+r_{\sigma_{1}(l)}^{1}}+\cdots+r_{\sigma_{2 k}(l)}^{2 k}
$$

with $0 \leq r_{l}^{i} \leq n-m$. Hence, by a computation similar to that of a complete Hankel hyperdeterminant,

$$
\prod_{l=1}^{m} M_{j_{\sigma_{1}(l)}^{1}+\cdots+j_{\sigma_{2 k}(l)}^{2 k}}=\prod_{l=1}^{m} M_{\lambda_{i}+s_{i}}
$$

with $k(i-1) \leq \lambda_{i} \leq k(m+i-2)$ and $0 \leq s_{i} \leq 2 k(n-m)$. And

$$
\operatorname{Det}(M[J])=\sum_{\tilde{\lambda}} c_{\tilde{\lambda}}^{n, k ; J} \prod_{i=1}^{m} \tilde{M}_{\tilde{\lambda}_{i}}
$$

where $k(i-1) \leq \tilde{\lambda}_{i} \leq k(2 n-m+i-2)$.

\section{Selberg's integral}

\subsection{From the Hankel form to the symmetric form}

Selberg's integral admits another hyperdeterminantal representation which can be obtained directly from the previous one (without manipulating the 
integral). It suffices to remark the following property

$$
\sum_{i=0}^{n}(-1)^{i}\left(\begin{array}{l}
n \\
i
\end{array}\right) \mathrm{B}(a+i, b)=\mathrm{B}(a, b+n) .
$$

Hence, we have from (refeq10)

$$
\mathbf{S}_{n}(a, b ; k)=(\overbrace{\mathrm{I}, \cdots, \mathrm{I}}^{k}, \overbrace{g, \cdots, g}^{k}) \cdot \mathbf{S}_{n}^{\text {Sym }}(a, b ; k)
$$

where $\mathrm{I}$ is the identity matrix, $g$ is the $n \times n$ matrix

$$
g=\sum_{1 \leq i, j \leq n}(-1)^{j}\left(\begin{array}{l}
i \\
j
\end{array}\right) \eta_{i} \otimes \eta_{j}
$$

and

$$
\mathbf{S}_{n}^{S y m}(a, b ; k)=\sum_{I, J \in\{0, \ldots, n-1\}^{k}} \mathrm{~B}(a+|I|, b+|J|) \eta_{I} \otimes \eta_{J}
$$

It follows from (12) that

$$
\operatorname{det}(g)^{k} \operatorname{Det}\left(\mathbf{S}_{n}^{\text {Sym }}(a, b ; k)\right)=\operatorname{Det}\left(\mathbf{S}_{n}(a, b ; k)\right)
$$

and, since

$$
\operatorname{det}(g)=(-1)^{\frac{n(n-1)}{2}},
$$

we find

$$
\operatorname{Det}\left(\mathbf{S}_{n}^{S y m}(a, b ; k)\right)=(-1)^{\frac{k n(n-1)}{2}} \operatorname{Det}\left(\mathbf{S}_{n}(a, b ; k)\right)
$$

without the help of the integral representation.

\subsection{Selberg's original proof}

Each step of Selberg's proof can be viewed as a manipulation on a hyperdeterminant (see 31 for example). It can be divided into two parts. In the first part, Selberg proved that

$$
\mathcal{S}_{n}(a, b ; k)=c_{n, k} \prod_{i=0}^{n-1} \frac{\Gamma(a+j k) \Gamma(b+j k)}{\Gamma(a+b+(n+j-1) k)} .
$$

where $c_{n, k}$ does not depend on $a$ and $b$. In this proof, Selberg started from the expansion of the Vandermonde determinant. In term of hyperdeterminants, it is equivalent to the expansion (29). For our purpose, equation (53) reads

$$
\operatorname{Det}\left(\mathbf{S}_{n}(a, b ; k)\right)=\alpha_{n, k} \prod_{i=0}^{n-1} \frac{\Gamma(a+j k) \Gamma(b+j k)}{\Gamma(a+b+(n+j-1) k)}
$$


where $\alpha_{n, k}=n ! c_{n, k}$. To obtain it, we start from formula (32)

$$
\operatorname{Det}\left(\mathbf{S}_{n}(a, b ; k)\right)=\sum_{\lambda} c_{\lambda}^{n, k} \mathrm{~B}\left(a+\lambda_{1}, b\right) \cdots \mathrm{B}\left(a+\lambda_{n}, b\right)
$$

where the sum is over all $n$-tuples $\lambda=\left(\lambda_{1}, \cdots, \lambda_{n}\right)$ such that $|\lambda|=k n(n-1)$ and for each $i \in\{1, \cdots, n\},(i-1) k \leq \lambda_{i} \leq k(n+i-2)$. Then, extracting common factors, we arrive at an expression of the form

$$
\operatorname{Det}\left(\mathbf{S}_{n}(a, b ; k)\right)=\frac{Q(a, b)}{R(b)} \prod_{i=0}^{n-1} \frac{\Gamma(a+j k) \Gamma(b+j k)}{\Gamma(a+b+(n+j-1) k)}
$$

where $Q(a, b)$ is a polynomial of degree at most $k n(n-1) / 2$ and $R(b)$ is a polynomial of degree $k n(n-1) / 2$. But (52) implies that

$$
\operatorname{Det}\left(\mathbf{S}_{n}(a, b ; k)\right)=\operatorname{Det}\left(\mathbf{S}_{n}(b, a ; k)\right)
$$

and then

$$
\frac{Q(a, b)}{R(b)}=\frac{Q(b, a)}{R(a)}
$$

which implies that $\alpha_{n, k}=\frac{Q(a, b)}{R(b)}$ is independent of $a$ and $b$.

The second part of Selberg's proof consists in establishing a recurrence relation for $\alpha_{n, k}$ :

$$
\alpha_{n, k}=\frac{(n k) !}{n !} \alpha_{n-1, k}
$$

This can be found from the limit

$$
L_{b ; k, n}=\lim _{a \rightarrow 0^{+}} a \operatorname{Det}\left(\mathbf{S}_{n}(a, b ; k)\right)
$$

Hence, one has

$$
L_{b ; k, n}=\lim _{a \rightarrow 0^{+}} \operatorname{Det}\left(\mathbf{T}_{a}\right)
$$

where $\mathbf{T}_{a}$ is the tensor defined by

$$
\begin{aligned}
\mathbf{T}_{a} & =\sum_{I \in\{0\} \times\{0, \ldots, n-1\}^{2 k-1}} a \mathrm{~B}(a+|I|, b) \eta_{I}+ \\
& +\sum_{I \in\{1, \cdots, n-1\} \times\{0, \ldots, n-1\}^{2 k-1}} \mathrm{~B}(a+I, b) \eta_{I}
\end{aligned}
$$

Remarking that

$$
\lim _{a \rightarrow 0^{+}} a \mathrm{~B}(a+n, b)=\delta_{n, 0}
$$


where $\delta_{i, j}$ is the Kronecker symbol, one obtains

$$
L_{b ; k, n}=\operatorname{Det}(\mathbf{T})
$$

where $\mathbf{T}$ is the tensor

$$
\mathbf{T}=\eta_{0} \otimes \cdots \otimes \eta_{0}+\sum_{I \in\{1, \cdots, n-1\} \times\{0, \ldots, n-1\}^{2 k-1}} \mathrm{~B}(|I|, b) \eta_{I}
$$

Hence, expanding by (22) with respect to the first subscript, one has

$$
L_{b ; k, n}=\operatorname{Det}\left(\mathbf{S}_{n-1}(1, b ; k)\right) .
$$

Together with (54) this equality gives the recurrence relation (59) and proves Selberg's identity.

\subsection{An alternative ending for Selberg's proof}

We shall now give a simpler proof of Selberg's identity by reducing it to the Dyson conjecture, which, as we have already seen, is by now a familiar and elementary statement involving no more than Lagrange interpolation. Using

the functional equation of the $\Gamma$ function and the linearity properties of the hyperdeterminant, we can recast the Hankel hyperdeterminantal expression of Selberg's integral in the form

$$
\operatorname{Det}\left(\mathbf{S}_{n}(a, b ; k)\right)=\mathrm{B}(a, b)^{n} \operatorname{Det}_{2 k}\left(\frac{(a)_{|I|}}{(a+b)_{|I|}}\right)_{0}^{n-1} .
$$

where $(a)_{n}=a(a+1) \cdots(a+n-1)$ denotes the Pochhammer symbol. The result will follow if we can obtain a closed form for the hyperdeterminant

$$
\mathcal{D}_{n, k}(a, b)=\operatorname{Det}_{2 k}\left(\frac{(a)_{|I|}}{(b)_{|I|}}\right)
$$

We start as in the previous proof, obtaining an analog of (54)

$$
\mathcal{D}_{k, n}(a, b)=\alpha_{n, k}^{\prime} \prod_{m=1}^{n} \frac{(a)_{k(m-1)}(b-a)_{k(m-1)}}{(b)_{k(n+m-1)}}
$$

where the constant $\alpha_{n, k}^{\prime}$ is independent of $a$ and $b$. Now, for every $a^{\prime}$

$$
\mathcal{D}_{k, n}(a, b)=\prod_{m=1}^{n} \frac{(a)_{k(m-1)}(b-a)_{k(m-1)}}{(b)_{k(n+m-1)}\left(a^{\prime}\right)_{k(m-1)}} \operatorname{Det}_{2 k}\left(\left(a^{\prime}\right)_{|I|}\right)_{0}^{n-1} \text {. }
$$


This means that Selberg's integral can be deduced from the knowledge of $\operatorname{Det}_{2 k}\left(\left(a^{\prime}\right)_{|I|}\right)_{0}^{n-1}$ for any particular choice of $a^{\prime}$. If we set $a^{\prime}=-k(n-1)$, one has by (34)

$$
\operatorname{Det}_{2 k}((-k(n-1))|I|)_{0}^{n-1}=(-1)^{k n(n-1)} \mathfrak{d}_{n, k}(k(n-1)) !^{n} .
$$

But $\mathfrak{d}_{n, k}$ has already been evaluated by means of the Dyson conjecture (39) and the closed form of Selberg's integral follows.

\section{Aomoto's integral}

\subsection{Another hyperdeterminantal representation}

On the integral representation (44), we obtain easily the following identity through the substitution $x_{i} \rightarrow 1-x_{i}$

$$
\mathcal{A}_{n}^{a, b ; k}(y)=(-1)^{n} \mathcal{A}_{n}^{b, a ; k}(1-y) .
$$

There is also a simple hyperdeterminantal proof of this assertion. We consider the tensor

$$
\mathbf{A}_{n}^{b, a ; k}(1-y)=\sum_{I \in\{0, \ldots, n-1\}^{2 k}}\left(1-y-\frac{b+|I|}{a+b+|I|}\right) \mathrm{B}(b+|I|, b) \eta_{I}
$$

and the action of $2 k$ copies of the matrix $g$ defined before. Using equality (46), one has

$$
(g, \ldots, g) \mathbf{A}_{n}^{b, a ; k}(1-y)=-\mathbf{A}_{n}^{a, b ; k}(y)
$$

Hence,

$$
\operatorname{Det}\left(\mathbf{A}_{n}^{a, b ; k}(y)\right)=(-1)^{n} \operatorname{Det}\left(\mathbf{A}_{n}^{b, a ; k}(1-y)\right) .
$$

\subsection{Minors of Selberg's hyperdeterminant}

To prove Aomoto's identity, we need some preliminary results on Selberg's hyperdeterminant. Let us consider a family of $2 k$ subsets $I=\left(I^{1}, \ldots, I^{2 k}\right)$ with $I^{j}=\left\{i_{0}^{j} \leq i_{1}^{j}, \ldots, \leq i_{m-1}^{j}\right\} \subset\{1, \cdots, n\}$ and the associated sub-tensor of Selberg's Hankel tensor:

$$
\mathbf{S}_{n}(a, b ; k)[I]=\sum_{0 \leq j_{1}, \ldots, j_{2 k} \leq m-1} \mathrm{~B}\left(a+i_{j_{1}}^{1}+\cdots+i_{j_{2 k}}^{2 k}, b\right) \eta_{i_{j_{1}}^{1}} \otimes \cdots \otimes \eta_{i_{j_{k}}^{2 k}}
$$

One can write by (45)

$$
\operatorname{Det}\left(\mathbf{S}_{n}(a, b ; k)[I]\right)=\sum_{\lambda} c_{\lambda}^{n, k ; I} \prod_{i=1}^{m} \mathrm{~B}\left(a+\lambda_{i}\right)
$$


where $k(i-1) \leq \lambda_{i} \leq k(2 n-m+i-2)$. Hence,

$$
\begin{array}{r}
\operatorname{Det}\left(\mathbf{S}_{n}(a, b ; k)[I]\right)=\prod_{i=1}^{m} \frac{\Gamma(a+k(i-1)) \Gamma(b)}{\Gamma(a+b+k(2 n-m+i-2))} \mathfrak{p}_{I}(a, b) \\
=\prod_{i=1}^{m} \frac{\Gamma(a+k(i-1)) \Gamma(b)}{\Gamma(a+b+k(2 n-i-1))} \mathfrak{p}_{I}(a, b)
\end{array}
$$

where $\mathfrak{p}_{I}(a, b)$ is a polynomial. In the same way, we will use the following identity

$$
\begin{aligned}
& \operatorname{Det}\left(\mathbf{S}_{n}(a+1, b ; k)[I]\right)=\prod_{i=1}^{n} \frac{1}{a+b+k(2 n-i-1)} \prod_{i=1}^{m}(a+k(i-1)) \times \\
& \times \prod_{i=m+1}^{n}(a+b+k(2 n-i-1)) \prod_{i=1}^{m} \frac{\Gamma(a+k(i-1)) \Gamma(b)}{\Gamma(a+b+k(2 n-i-1))} \mathfrak{p}_{I}(a+1, b) .
\end{aligned}
$$

which follows immediately from (78) $)$.

\subsection{A proof of Aomoto's identity}

We start from the hyperdeterminantal representation and we remark that

$$
\mathbf{A}_{n}^{a, b ; k}(y)=\mathbf{S}(a, b ; k) y-\mathbf{S}(a+1, b ; k) .
$$

From (19), we obtain

$$
\begin{array}{r}
\operatorname{Det}\left(\mathbf{A}_{n}^{a, b ; k}(y)\right)=\sum_{r=0}^{n}(-1)^{n-r} y^{r} \times \\
\times \sum_{(I, J) \in \mathfrak{C}_{n, k}^{r}} \epsilon(I, J) \operatorname{Det}(\mathbf{S}(a, b ; k)[I]) \operatorname{Det}(\mathbf{S}(a+1, b ; k)[J]) .
\end{array}
$$

From (178) and (79), one has

$$
\begin{array}{r}
\operatorname{Det}\left(\mathbf{A}_{n}^{a, b ; k}(y)\right)=\prod_{i=1}^{n} \frac{1}{a+b+k(2 n-i-1)} \times \\
\times \sum_{r=0}^{n} y^{r} \prod_{i=1}^{n} \frac{\Gamma(a+k(i-1)) \Gamma(b)}{\Gamma(a+b+k-2 n-i-1)} \times \\
\times \prod_{i=1}^{n-r}(a+k(i-1)) \prod_{i=n-r+1}^{n}(a+b+k(2 n-i-1)) \mathfrak{P}_{r}(a, b)
\end{array}
$$


where $\mathfrak{P}_{r}(a, b)$ is a polynomial in $a$ and $b$.

On the other hand, we expand the hyperdeterminantal representation of Aomoto's integral by (32) and obtain, after extracting common factors as above,

$$
\begin{aligned}
\operatorname{Det}\left(\mathbf{A}_{n}^{a, b ; k}\right)= & \operatorname{Det}\left(\mathbf{S}_{n}(a, b ; k)\right) \sum_{\lambda} \frac{c_{\lambda}^{n, k}}{Q(b)} \prod_{i=1}^{n}\left\{y(a)_{k(i-1)}^{\lambda_{i}-1}(a+b)_{\lambda_{i}}^{k(n+i-2)-1}\right. \\
& \left.-(a)_{k(i-1)}^{\lambda_{i}}(a+b)_{\lambda_{i}+1}^{k(n+i-2)-1}\right\}
\end{aligned}
$$

where $Q(b)$ is a polynomial of degree $k n(n-1)$ in $b,(a)_{n}^{m}$ denotes $(a+n)(a+$ $n+1) \cdots(a+m)$ if $n \leq m$ and $(a)_{n}^{m}=1$ otherwise and the sum is over the partitions $\lambda=\left(\lambda_{1} \leq \cdots \leq \lambda_{n}\right)$ verifying $k(i-1) \leq \lambda_{i} \leq k(n+i-1)$ for each $i \in\{1, \cdots, n\}$. After combining the coefficients of the $y^{i}$ s, one obtains

$$
\operatorname{Det}\left(\mathbf{A}_{n}^{a, b ; k}(y)\right)=\operatorname{Det}\left(\mathbf{S}_{n}(a, b ; k)\right) \sum_{r=0}^{n} y^{r} \frac{\mathfrak{Q}_{r}(a, b)}{Q(b)}
$$

where $\mathfrak{Q}_{r}(a, b)$ is a polynomial. Hence, comparing the coefficients of $y^{i}$ in the expressions (82) and (84), we find

$$
\begin{gathered}
\operatorname{Det}\left(\mathbf{A}_{n}^{a, b ; k}(y)\right)=\operatorname{Det}\left(\mathbf{S}_{n}(a, b ; k)\right) \prod_{i=0}^{n-1} \frac{1}{a+b+k(n+i-1)} \times \\
\times \sum_{i=0}^{n} y^{i} \frac{P_{i}(a, b)}{Q(b)} \prod_{j=1}^{n-i}(a+k(n-j)) \prod_{j=n-i+1}^{n}(a+b+k(2 n-j-1))
\end{gathered}
$$

where $P_{i}(a, b)$ is a polynomial. Remark that each $P_{i}(a, b)$ is of degree in $b$ at most $k n(n-1)$. Now, we apply (75) and we equate the coefficients of $y^{i}$ in the left and right hand sides. After simplification, one obtains for each $i$

$$
\left.\sum_{j=i}^{n}\left(\begin{array}{l}
j \\
i
\end{array}\right) P_{j}(b, a) F_{j}(b, a) Q(b)=(-1)^{n-i} P_{i}(a, b) F_{i}(a, b) Q(a)\right)
$$

where

$$
F_{i}(a, b)=\prod_{m=1}^{n-i}(a+k(n-m)) \prod_{m=n-i+1}^{n}(a+b+k(2 n-m-1)) .
$$

Remarking that $Q(b)$ does not divide $F_{i}(a, b)$, equality (866) implies

$$
P_{i}(a, b)=R_{i}(a) Q(b) \text {. }
$$


Hence, setting as in the previous section, $a=-k(n-1)$ in

$$
\sum_{j=i}^{n}\left(\begin{array}{l}
j \\
i
\end{array}\right) R_{j}(b) F_{j}(b, a)=(-1)^{n-i} R_{i}(a) F_{i}(a, b)
$$

and remarking that

$$
F_{i}(-k(n-1), b)=0
$$

and

$$
F_{i}(b,-k(n-1))=\prod_{m=1}^{n}(b+k(n-m)),
$$

one obtains the recurrence relation

$$
R_{i}(b)=-\sum_{j=i+1}^{n}\left(\begin{array}{l}
j \\
i
\end{array}\right) R_{j}(b) .
$$

The starting point of the induction is $R_{n}(b)=\frac{1}{n !}$, which follows from the fact that the coefficient of $y^{n}$ in the expansion of the hyperdeterminant is the value of Selberg's integral. Solving the recurrence relation, one finds that each $R_{i}(b)$ is in fact independent of $b$, and that

$$
R_{i}(b)=\frac{(-1)^{n-i}}{n !}\left(\begin{array}{c}
n \\
i
\end{array}\right)
$$

Hence,

$$
\begin{gathered}
\operatorname{Det}\left(\mathbf{A}_{n}^{a, b ; k}(y)\right)=\operatorname{Det}\left(\mathbf{S}_{n}(a, b ; k)\right) \frac{1}{n !} \prod_{i=0}^{n-1} \frac{1}{a+b+k(n+i-1)} \times \\
\times \sum_{i=0}^{n}(-1)^{n-i}\left(\begin{array}{c}
n \\
i
\end{array}\right) \prod_{m=1}^{n-i}(a+k(n-m)) \prod_{m=n-i+1}^{n}(a+b+k(2 n-m-1)) y^{i}(94) \\
=(-1)^{n} \operatorname{Det}\left(\mathbf{S}_{n}(a, b ; k)\right) \frac{\left(\frac{a}{k}\right)_{n}}{n !\left(\frac{a}{k}+\frac{b}{k}+n-1\right)_{n}} \sum_{i=0}^{n} \frac{(-n)_{i}\left(\frac{a}{k}+\frac{b}{k}+n-1\right)_{i}}{\left(\frac{a}{k}\right)_{i}} \frac{y^{i}}{i !} .
\end{gathered}
$$

We recognize a hypergeometric function of type ${ }_{2} F_{1}$ which can be evaluated as a monic Jacobi polynomial (see [2] for details)

$$
\begin{aligned}
\operatorname{Det}\left(\mathbf{A}_{n}^{a, b ; k}(y)\right)= & (-1)^{n} \operatorname{Det}\left(\mathbf{S}_{n}(a, b ; k)\right) \frac{\left(\frac{a}{k}\right)_{n}}{n !\left(\frac{a}{k}+\frac{b}{k}+n-1\right)_{n}} \times \\
& \times{ }_{2} F_{1}\left(\begin{array}{c}
\left.-n, n+\frac{a}{k}+\frac{b}{k}-1 \mid y\right) \\
\frac{a}{k}
\end{array}\right. \\
= & \frac{(-2)^{-n}}{n !} \operatorname{Det}\left(\mathbf{S}_{n}(a, b ; k)\right) P_{n}^{\frac{a}{k}-1, \frac{b}{k}-1}(1-2 y)
\end{aligned}
$$

Aomoto's equality is therefore proved. 


\section{Conclusion}

The examples discussed in this article show that the hyperdeterminantal calculus is a pertinent tool to handle an interesting class of multiple integrals. The hyperdeterminant is a particular invariant of hypermatrices. In the case of antisymmetric hypermatrices, another invariant has similar properties: the hyperpfaffian [5, 27]. In the classical case of matrices, de Bruijn has shown in [1] that multiple integrals of some determinants could be evaluated as Pfaffians. In [27, we have found some generalizations to hyperpfaffians of these identities. In fact, the generalized Heine theorem is a particular case of the generalized de Bruijn integral, where the determinant can be factored into a product of determinants of matrices whose dimension is the number of integration variables. There exist other invariant polynomials of hypermatrices [8, 9, 10, 17] which can be, in principle, computed using Cayley's Omega process or other methods of invariant theory (see [29, 6, 7 ] for examples involving $2 \times 2 \times 2 \times 2$ and $3 \times 3 \times 3$ hypermatrices). A natural question is whether there exist other integral identities involving some of them.

Other generalizations of Selberg integral are encountered in the physical litterature. For example the Dotsenko-Fateev (see [12]) and Kaneko integrals (see [20]) give the partition functions of various systems.

Kaneko's integral reads

$$
K_{n, \gamma}^{a, b}\left(y_{1}, \cdots, y_{m}\right)=\int_{0}^{1}|\Delta(x)|^{2 \gamma} \prod_{i=1}^{n}\left(x_{i}^{a-1}\left(1-x_{i}\right)^{b-1} \prod_{j=1}^{n}\left(x_{i}-y_{j}\right) d x_{i}\right)
$$

and is evaluated in terms of generalized orthogonal polynomials associated with Jack polynomials (see [23, 24, 25]). By the generalized Heine theorem, when $\gamma$ is an integer, Kaneko's integral can be evaluated as a hyperdeterminant of moments (see 28]). Kaneko's proof of his identity is related to the Calogero-Sutherland model. Up to now, we have been unable unable to find a hyperdeterminantal interpretation of this. In the same way, Anderson's (see [1]) and Aomoto's (see [3]) proofs of Selberg's integral seem to contain information unrelated to the hyperdeterminantal representations.

\section{References}

[1] Anderson, G.W., 1987, Quart. J. Math. Oxford, 38, 385-399.

[2] Andrews, G.E., Askey, R., and Roy, R., 1999, Special Functions, (Encyclopedia of Mathematics and its applications 71, Cambridge University Press).

[3] Aomoto, K., 1987, SIAM J. Math. Anal., 18, 545-549. 
[4] Barsky, D., and Carpentier, M., 1996, Electronic J. Combinatorics 3, R1.

[5] Barvinok, A.I., 1995, Math. Program., 69, 449-470.

[6] Briand, E., Luque, J.-G., and Thibon, J.-Y., 2003, J. Phys. A: Math. Gen. 36, 99159927.

[7] Briand, E., Luque, J.-G., Thibon, J.-Y., and Verstraete, F., 2003, arXiv:quant-ph/0306122

[8] Cayley, A., 1843, Trans. Cambridge Phil. Soc. VIII, 1-16.

[9] Cayley, A., 1846, J. reine und angew. Math., 30, 1-37.

[10] Cayley, A., 1851, Cambridge and Dublin Math. Journal VII, 40-51.

[11] de Bruijn, N.G., 1956, J. Indian Math. Soc. (N.S.), 19, 133-151.

[12] Dotsenko, V.S., and Fateev, V.A., 1985, Nucl. Phys. B, 251, 691-734.

[13] Dyson, F.J., 1962, J. Math. Phys., 3, 140-156.

[14] Forrester, P.J., 1998, Random matrices, log-gases and the Calogero-Sutherland model, in Quantum Many-Body Problems and Representation theory (MSJ Mem. Vol1) (Tokyo: Mathematical Society of Japan), 97-181.

[15] Forrester, P.J., 1993, Nucl. Phys. B, 402, 709-728.

[16] Forrester, P.J., 1992, Phys. Lett. A, 163, 121-126.

[17] Gelfand, I.M., Kapranov, M.M., and Zelevinsky, A.V, 1994, Discriminants, Resultants and Multidimensional Determinants, (Mathematics: Theory \& Applications. Birkhuser Boston, Inc., Boston, MA).

[18] Good, I.J., 1970, J. Math. Phys, 11, 1884-1886.

[19] Heine, H.E., 1878, 1881, Handbuch der Kugelfunktionen, 2 vol., (2nd edition, Berlin).

[20] Kaneko, J., 1993, SIAM J. Math. Anal., 24, 1086-1110.

[21] King, R.C., Toumazet, F., and Wybourne, B.G., 2003, preprint, http://www.phys.uni.torun.pl/ bgw/vanp.ps

[22] Krattenthaler, C., 1998, The Andrews Festschrift (Séminaire Lotharingien de Combinatoire, 42) 67pp (electronic).

[23] Lassalle, M., 1991, C.R. Acad. Sci. Paris, ser. I, 312, 425-428.

[24] Lassalle, M., 1991, C.R. Acad. Sci. Paris, ser. I, 312, 725-728.

[25] Lassalle, M., 1991, C.R. Acad. Sci. Paris, ser. I, 313, 579-582.

[26] Laughlin, R.B., 1983, Phys. Rev. Lett., 50, 1395-1398.

[27] Luque, J.-G., and Thibon, J.-Y., 2002, Adv. Appl. Math., 29, 620-646.

[28] Luque, J.-G., and Thibon, J.-Y., 2003, J. Phys. A.: Math. Gen., 36, 5267-5292.

[29] Luque, J.-G., and Thibon, J.-Y., 2003, Phys. Rev. A, 67, 042303, 1-5.

[30] Macdonald, I.G., 1995, Symmetric functions and Hall polynomials, 2nd ed., (Oxford: Clarendon press). 
[31] Mehta, M.L., 1991, Random matrices, 2nd ed., (Academic Press).

[32] Scharf, T., Thibon, J.-Y., and Wybourne, B.G., 1994, J. Phys. A.: Math. Gen., 27, 4211-4219.

[33] Selberg, A. 1944, Normat., 26, 71-78.

[34] Sokolov, N.P., 1960, Spatial matrices and their applications, (Gosudarstv. Izdat. Fiz.Math.Lit., Moscow), in Russian.

[35] Sokolov, N.P., 1972, Introduction to the Theory of Multidimensional Matrices, (Kiev: Nukova Dumka), in Rusian.

[36] Titchmarsch, E.C., 1939, Theory of functions, (Oxford University Press, London and New-York).

[37] Wigner, E.P., 1951, Proc. Cambridge Phil. Soc., 47, 790-798.

[38] Wilson, K.G., 1962, J. Math. Phys, 3, 1040-1043.

[39] Wybourne, B.G., 2002, The Vandermonde Determinant Revisited, in SSPCM'2002, Myczkowce, Poland, (Singapore: World Scientific), 74-87.

[40] Wybourne, B.G., 2003, preprint http://www.phys.uni.torun.pl/ $\sim$ bgw/bgwybourne.ps, 2003. 